\title{
The Exact Hausdorfi Measure of the Zero Set of a Stable Process
}

\author{
S. J. TAYLOR and J. G. WENDEL *
}

Received May 20, 1966

\section{Introduction}

In this paper we continue the study of the zero set of a stable process on the line. In [20] one of us showed that the zero set of the Wiener process has Hausdorff dimension $1 / 2$ and zero $\Lambda^{1 / 2}$-measure, with probability 1 . This result was partially extended by BLUmenthal and Geroon [2] to the symmetric stable process of index $\alpha>1$; they showed that in this case the zero set has dimension $\beta=1-1 / \alpha$. There is of course no problem when $\alpha \leqq 1$, for then the zero set is almost surely trivial.

In 1957 Paul LÉvy suggested that for the Wiener process it should be possible to determine an exact measure function for the zero set $Z=\{t: X(t)=0\}$. By this is meant a function $\varphi(h)$ defined for small $h \geqq 0$, vanishing at the origin, increasing and continuous, such that the random quantities

$$
\varphi-m(Z \cap[0, t]), \quad t>0,
$$

are almost surely positive and finite. Here $\varphi-m(E)$ denotes the Hausdorff $\varphi$-measure of the set $E$; the definition is recalled in section 5 . The main objectives of the present investigation are to find such a measure function $\varphi$ for a general (not necessarily symmetric) stable process $X(l)$ of index $\alpha>1$, and to identify the resulting stochastic process as defined by (1). In fact we show that this stochastic process, which should be thought of as measuring the extent of the zero set in the time interval $[0, t]$, can be identified, apart from a constant of proportionality, with the local time at zero in the sense of BoyLAN [4] or BLUMENTHAT and GETOoR [3]; cf. section 3 below. To formulate this precisely let us state our main result.

Theorem 1. Suppose $X(t)$ is a stable process of order $\alpha>1$ on the line, with zero set $Z$ and local time at zero $A(t)$. Then there is a finite positive constant $c_{1}$ depending on the parameters of the process such that almost surely

$$
p-m(Z \cap[0, t])=c_{1} A(t)
$$

for all $t>0$, where $\varphi(h)=h^{\beta}(\log |\log h|)^{1-\beta}, \beta=1-1 / \alpha$.

The plan of the paper is as follows. Section 2 contains the basic definitions and asymptotic relations needed in the sequel. In section 3 we review properties of the local time and of its inverse function $\tau(t)$. Local asymptotic laws for $\tau$ and $A$ are derived in section 4, and their counterparts at infinity are stated, generalizing a result of Kesten [11] for the Wiener local time. Methods of Cimselski and TAYLOR [5] are modified in section 5 to obtain a positive lower bound for the

* During the preparation of this paper the authors received partial support from the National Science Foundation, under contracts GP4153 and GP3466. 
$\varphi$-measure of $Z$; a finite upper bound is obtained in section 6 , by a method similar to that in [21]. The paper concludes with the proof of the main theorem in section 7 . Positive constants whose value is unimportant occur frequently: these will be denoted by $c_{1}, c_{2}, \ldots, c_{25}$.

\section{Preliminaries}

Throughout this paper we will be considering versions of stable processes on the real line which satisfy HuNT's hypotheses $(A)$ : they are strongly Markovian, and have right-continuous and quasi-left-continuous paths almost surely. (The reader unfamiliar with HuNT's contribution [9] will find an admirable summary of the meaning of these conditions in GETOOR [8].) The general stable process of index $\alpha$, see Lívy [14], is a process $X(t)=X(t, \omega)$ starting at the origin, having stationary independent increments, and for which the characteristic function $E\{\exp i \theta X(t)\}$ is $\exp \{-t y(\theta)\}$ where, for $0<\alpha<1$ or $1<\alpha \leqq 2$,

$$
\psi(\theta)=a|\theta| \alpha\{1+i \gamma(\operatorname{sgn} \theta) \tan (\pi \alpha / 2)\},
$$

in which $\alpha>0,-1 \leqq \gamma \leqq 1$. If $\gamma=0$ then $X(t)$ is symmetric. When $\alpha=2$ we may as well take $\gamma=0$ in (2); then $X(t)$ is the Wiener process. When $\alpha=1$ it is necessary to modify (2), but the stable process of index 1 will play no role in our discussion. In fact from this point on we will assume that we are discussing a fixed process $X(t)$ with index $\alpha>1$. It is well known that in this case the process is point recurrent, so that the zero set $Z$ is unbounded almost surely.

If in (2) we have $\gamma=-1$ and $0<\alpha<1$ the corresponding process has nonnegative increments and is called a stable subordinator. To distinguish this case we will denote a stable subordinator by $\tau(t)$ and call its index $\beta, 0<\beta<1$. The range of a stable subordinator is the random point set

$$
Q=\{\tau(t): 0 \leqq t<\infty\} .
$$

Whenever $X$ and $\tau$ occur together in a discussion it is understood that $\beta=1-1 / \alpha$. The Laplace transform of the distribution of $\tau(t)$ is given by

$$
E\{\exp (-s \tau(t))\}=\exp \left(-b t s^{\beta}\right)
$$

where $b$ is a positive constant. Note that since $\tau(t)$ is increasing, the closure $\bar{Q}$ of the range differs from $Q$ only in the countable set of left limits $\tau(t-0)$ at points of discontinuity.

We require estimates for the tails of the distribution of $\tau(1)$, which we collect now for ease of reference; these are stated for the case $b=1$, the corresponding results for general $b$ being obtainable by a scale change. Let $F(x)=P\{\tau(1) \leqq x\}$ be the distribution function of $\tau(1)$. Then

$$
F(x) \sim c_{2} x^{\beta / 2(1-\beta)} \exp \left(-c_{3} x^{-\beta /(1-\beta)}\right), \quad x \rightarrow 0,
$$

with $c_{2}=\left(2 \pi c_{3}\right)^{1 / 2}, c_{3}=(1-\beta) \beta^{1-\beta} ;$ and

$$
1-F(x) \sim c_{4} x^{-\beta}, \quad x \rightarrow \infty,
$$

where $c_{4}=1 / \Gamma(1-\beta)$.

The relations (4) and (5) are easily deduced from the corresponding results for the density $F^{\prime}(x)$, due respectively to LINNIK [14] and PoLLard [16]; cf. also the convenient tabulation in SKoROHOD [18].

The following lemma is due to DYNKIN [6]. 
Lemma 1. The probability that an interval $[a, b)$ contains a point of $Q$ is equal to

where $c_{5}=\pi^{-1} \sin \pi \beta$.

$$
c_{5} \int_{1}^{b / a} u^{-1}(u-1)^{-\beta} d u
$$

\section{The Local Time and its Inverse Function}

In [2] BuUMENTHAL and GeToor generalized a result of LÉvy by showing that $\bar{Z}$ and $\bar{Q}$, the closures of $Z$ and $Q$, are stochastically the same in the following sense: if $\left\{I_{k}\right\}, k=1,2, \ldots, n$, is any finite disjoint collection of open intervals then the two events

and

$$
\left\{\bar{Z} \cap I_{k} \neq \emptyset, k=1,2, \ldots, n\right\}
$$

$$
\left\{\bar{Q} \cap I_{k} \neq \emptyset, k=1,2, \ldots, n\right\}
$$

have the same probability. This equality of probability then extends to the $\sigma$-algebra generated by such events, but it is not always technically easy to check that a given event of interest belongs to this $\sigma$-algebra. This complication is circumvented and the stochastic identity of the two random sets is neatly accounted for by the theory of local times, which we now proceed to sketch.

It was shown by TroTrer [22] for the Wiener process, and by BorLAN [4] for a wide class of processes including our $X(t)$, that with probability one there is a function $L(t, x)$, called the local time at $x$, which serves as a density function for the occupation times of the process. That is, for each sample path outside a fixed null set the equation

$$
\int_{0}^{t} I_{B}(X(u)) d u=\int_{B} L(t, x) d x
$$

holds for every Borel set $B$ and each $t>0$. The function $L$ is jointly continuous in both arguments and non-decreasing in $t$. (Applying the machinery of additive functionals of Markov processes BLUMENTHAL and GETOOR [3] have developed a similar and in some ways more extensive theory of local times. Neither they nor we are concerned with the dependence of the local time on the space variable $x$, and it is not difficult to verify that Borlan's $L(t, 0)$ and BLumenthaL-Getoon's $A(t)$ may be identified.) STowe [19] then showed that the function inverse to $A(t)$, namely

$$
\tau(t)=\tau(t, \omega)=\inf \{u: A(u, \omega)>t\},
$$

is a stable subordinator of index $\beta=1-1 / \alpha$ and scale factor $b$ given by

$$
b=\alpha\left(\sin \pi \alpha^{-1}\right) a^{1 / \alpha}\left[\operatorname{Re}\left\{\left(1+i \gamma \tan (\pi \alpha / 2)^{-1 / \alpha}\right\}\right]^{-1} .\right.
$$

(It should be clear that all of the functions $X(t, \omega), \tau(t, \omega), A(t, \omega)$, are defined on a single probability space $\Omega$ of points $\omega$.) He showed further that $\widetilde{Q}$, the closure of the range of $\tau$, is almost surely equal to the zero set $Z$, which in turn is almost surely a closed set, the set of points of increase of $A(t)$. These results are valid for a slightly larger class of Markor processes (which STONE calls ,semi-stable") that need not have independent increments; in particular all stable subordinators of index $\beta<1$ appear as inverses of appropriate local times, whereas only those for 
which $\beta \leqq 1 / 2$ arise from our $X(t)$, since then $\beta=1-1 / \alpha$ and $\alpha \leqq 2$. Our results, depending as they do on those of STONE, are thus valid for the zero sets of semistable processes; in the definition of the exact measure function $\varphi$ we have only to take $\beta$ as the primary parameter.

This means that we can consider the subordinator $\tau(t)$ as the primary process, so that $A$ becomes the "hitting time" process for $\tau$, given by

$$
A(u)=A(u, \omega)=\inf \{t: \tau(t)>u\}
$$

and we may as well study $\varphi-m(Q \cap[0, t])$ instead of $(1)$, since $\bar{Q}-Q$ is countable and $Z=\bar{Q}$. It is clear from (3) that a change in $b$ amounts only to a change in the time scale of the subordinator, and therefore does not affect the range $Q$. Then it is a consequence of STONE's results that the stochastic structure of the zero set $Z$ does not depend on the parameters $a, \gamma$. Hence it will be enough for us to prove Theorem 1 in the case $b=1$; the general case will follow on replacing $c_{1}$ by $b c_{1}$.

\section{Local Asymptotic Results}

Our main object in this section is to obtain an asymptotic law for the subordinator $\tau(t)$ as $t \rightarrow 0$. Although the result of the next theorem is an immediate corollary of results recently obtained by FRISTEDT [7] we present a proof, as we need some auxiliary estimates not contained in [7].

Theorem 2. If $\tau(t)$ is a stable subordinator of index $\beta, 0<\beta<1, b=1$, and $\eta(t)=t^{1 / \beta}(\log |\log t|)^{1-1 / \beta}$ then with probability 1

$$
\liminf _{t \rightarrow 0} \tau(t) / \eta(t)=d,
$$

where $d=c_{3}^{(1-\beta) / \beta}$.

Proof. We first show that the lim inf is almost surely at least $d$. Given a positive $\lambda<1$ choose $q$ between $\lambda^{\beta}$ and 1 , and set $t_{k}=q^{k}, k=1,2, \ldots$ Since $\tau(t)$ and ultimately $\eta(t)$ decrease with $t$ we have, for $t_{k+1} \leqq t \leqq t_{k}$ and $k$ large,

$$
\tau(t) / \eta(t) \geqq \tau\left(t_{k+1}\right) / \eta\left(t_{k}\right) .
$$

Let $A_{k}$ be the event that the right member of (6) is less than $\lambda d$. Since the distributions of $\tau(t)$ and $t^{1 / \beta} \tau(1)$ coincide we have

$$
\begin{aligned}
P\left\{A_{k}\right\} & =P\left\{\tau(1)<\lambda d t_{k+1}^{-1 / \beta} \eta\left(t_{k}\right)\right\} \\
& =F\left(q^{-1 / \beta} \lambda d\{\log k+\log |\log q|\}^{1-1 / \beta}\right) \\
& \sim c_{6}(\log k)^{c_{2}} \exp \left(-c_{8} \log k\right), \quad k \rightarrow \infty .
\end{aligned}
$$

Here $c_{6}, c_{7}$ are unimportant constants, while $c_{8}=(q \lambda-\beta)^{1 /(1-\beta)}>1$. Therefore $\sum_{k} P\left(A_{k}\right)<\infty$. In view of $(6)$ and the arbitrariness of $\lambda<1$ the Borel-Cantelli lemma then implies that $\liminf _{t \rightarrow 0} \tau(t) / \eta(t) \geqq d$.

The opposite inequality could now be easily deduced from Borel-Cantelli arguments applied to the right member of the inequality

$$
\lim \inf \tau\left(t_{k}\right) / \eta\left(t_{k}\right) \leqq \lim \inf \left\{\tau\left(t_{k}\right)-\tau\left(t_{k+1}\right)\right\} / \eta\left(t_{k}\right)+\lim \sup \tau\left(t_{k+1}\right) / \eta\left(t_{k}\right)
$$


for $t_{k}$ tending sufficiently rapidly to zero, say $t_{k}=\exp \left(-k^{1+\delta}\right), \delta>0$. However, we need an upper bound for the probability of events of the form

$$
\{\tau(t)>(1+2 \varepsilon) d \eta(t) \text { for all } t \in(l, m)\}
$$

where $0<l<m$ and $l$ is much smaller than $m$. To this end consider the events

$$
D_{k}=\left\{\tau\left(t_{k}\right)>(1+2 \varepsilon) d \eta\left(t_{k}\right)\right\}, t_{k}=\exp \left(-k^{1+\delta}\right), \delta>0, k=1,2, \ldots
$$

Lemma 2. For $\varepsilon>0$ there exist positive constants $\delta, c_{15}, c_{16}$, and $m_{0}$ such that

$$
P\left\{\bigcap_{k=m}^{2 m} D_{k}\right\} \leqq \exp \left(-c_{15} m^{c_{16}}\right), \quad m \geqq m_{0} .
$$

Proof. Let $E_{k}=\left\{\tau\left(t_{k}\right)-\tau\left(t_{k+1}\right)>(1+\varepsilon) d \eta\left(t_{k}\right)\right\}, \quad P\left\{E_{k}\right\}=1-p_{k}$, $F_{k}=\left\{\tau\left(t_{k+1}\right)>\varepsilon d \eta\left(t_{k}\right)\right\}, P\left\{F_{k}\right\}=q_{k}$. Since $\bigcap D_{k} \subseteq \bigcap E_{k} \cup \bigcup F_{k}$ and the events $E_{k}$ are independent it follows that

$$
\begin{aligned}
P\left\{\bigcap D_{k}\right\} & \leqq \prod\left(1-p_{k}\right)+\sum q_{k} \\
& \leqq \exp \left(-\sum p_{k}\right)+\sum q_{k} .
\end{aligned}
$$

Now we have

$$
\begin{aligned}
p_{k} & =F\left(\left(t_{k}-t_{k+1}\right)^{-1 / \beta}(1+\varepsilon) d \eta\left(t_{k}\right)\right) \\
& =F^{\prime}\left(\left(1-t_{k}^{-1} t_{k+1}\right)^{-1 / \beta}(1+\varepsilon) d\left\{\log \left|\log t_{k}\right|\right\}^{1-1 / \beta}\right) \\
& \geqq F^{\prime}\left((1+\varepsilon) d\left\{\log (2 m)^{\delta+1}\right\}^{1-1 / \beta}\right)
\end{aligned}
$$

for $k \leqq 2 m$, since $t_{k}>t_{k+1}$ and $1-1 / \beta<0$. Hence, using (4),

$$
\sum_{m}^{2 m} p_{k} \geqq m c_{9}(\log m)^{c_{11}} \exp \left\{-(1+\varepsilon)^{-\beta /(1-\beta)}(\delta+1) \log (2 m)\right\} \geqq c_{9}(\log m)^{c_{11}} m^{c_{10}}
$$

for $m$ sufficiently large; positivity of the constant $c_{10}$ is assured by taking $\delta$ sufficiently small. The logarithmic factor may be absorbed by making a small downward adjustment of $c_{10}$. This leads to an estimate of the form

$$
\exp \left(-\sum_{m}^{2 m} p_{k}\right)<\exp \left(-c_{9} m^{c_{12}}\right), m \text { large . }
$$

Similarly, using (5), we have

$$
\begin{aligned}
q_{k} & =1-F\left(t_{k+1}^{-1 / \beta} \varepsilon d \eta\left(t_{k}\right)\right) \\
& =1-F\left(\varepsilon d\left(t_{k} / t_{k+1}\right)^{1 / \beta}\left\{\log \left|\log t_{k}\right|\right\}^{1-1 / \beta}\right) \\
& \leqq c_{13}\left(t_{k+1} / t_{k}\right)\left(\log \left|\log t_{k}\right|\right)^{1-\beta} \\
& \leqq c_{13}\left(\log k^{\delta+1}\right)^{1-\beta} \exp \left(-(\delta+1) k^{\delta}\right) \\
& \leqq c_{14}(\log 2 m)^{1-\beta} \exp \left(-(\delta+1) m^{\delta}\right)
\end{aligned}
$$

for $m \leqq k \leqq 2 m$. Then

$$
\sum_{m}^{2 m} q_{k} \leqq c_{14}(m+1)(\log 2 m) \exp \left(-(\delta+1) m^{\delta}\right), m \text { large } .
$$

The factors outside the exponential may be absorbed into the exponent, with small adjustments. Then the sum of the two bounds (8) and (9) is majorized by another of the desired form, for all sufficiently large $m$. Reference to (7) completes the proof of Lemma 2 . 
Corollary. If $M \geqq 2 m$ and $m \geqq m_{0}$ then

Proof. $\bigcap_{m}^{M} D_{k} \subseteq \bigcap_{[M / 2]}^{M} D_{k}$.

$$
P\left\{\bigcap_{m}^{M} D_{k}\right\} \leqq \exp \left(-c_{17} M^{c_{16}}\right) .
$$

It follows from the corollary that $P\left\{\lim\right.$ inf $\left.D_{k}\right\}=0$, and therefore that for each $\varepsilon>0, P\{\liminf \tau(t) / \eta(t) \geqq(1+2 \varepsilon) d\}=0$. This completes the proof of Theorem 2.

Similar calculations show that the theorem also holds for large $t$ :

Theorem 3. Under the conditions of Theorem 2 ,

$$
P\left\{\liminf _{t \rightarrow \infty} \tau(t) / \eta(t)=d\right\}=1
$$

However, the slow decay of $1-F(x)$ as $x \rightarrow \infty$ (cf. (5)) makes it apparent that there can be no nondegenerate "lim sup" law for $\tau(t), t$ tending to zero or infinity. It is possible to define upper and lower classes of functions and to characterize them using results of KHINCHIN [12]; see FrISTEd [7] for a summary.

Since $\tau$ and $A$ are inverse to each other, while $\eta$ and $\varphi$ are asymptotically so (i. e. $\varphi(\eta(t)) \sim t \sim \eta(\varphi(t)))$ it is possible to restate Theorems 2 and 3 in terms of the local time $A$ and the function $\varphi$.

Theorem 4. $P\left\{\lim _{t \rightarrow 0, \infty} \sup A(t) / \varphi(t)=d^{-\beta}\right\}=1$.

This generalizes Theorem 1 of KEsTEN [11] for the Wiener process. Note that the remark after Theorem 3 implies that there cannot be any result of the form

$$
\liminf _{t \rightarrow \infty} A(t) / h(t)=c, \quad 0<c<\infty .
$$

We have not attempted to extend KESTEN's Theorem 2, to give a strong law for

$$
\liminf _{t \rightarrow \infty}\left\{\sup _{x} L(t, x)\right\} / h(t) \text {. }
$$

For convenience of reference we now give a special case of the corollary framed in terms of $A$ and $\varphi$. events

Lemma 3. Let $t_{k}=\exp \left(-k^{1+\delta}\right), u_{k}=2 d \eta\left(t_{k}\right)$ and $d^{\prime}=(2 d)^{-\beta}$. Define the

$$
D_{k}^{\prime}=\left\{A\left(u_{k}\right)<d^{\prime} \varphi\left(u_{k}\right) / 2\right\} .
$$

Then for appropriate positive constants $\delta, c_{16}, c_{17}$ and $m_{0}$ we have

$$
P\left\{\bigcap_{m}^{M} D_{k}^{\prime}\right\} \leqq \exp \left(-c_{17} M^{c_{16}}\right)
$$

providing that $m \geqq m_{0}$ and $M \geqq 2 m$.

Proof. For sufficiently large $k$ we have $d^{\prime} \varphi\left(u_{k}\right) / 2 \leqq \eta^{-1}\left(u_{k} / 2 d\right)=t_{k}$. Then $D_{k}^{\prime}$ is contained in the event $\left\{A\left(u_{k}\right)<t_{k}\right\}=\left\{u_{k}<\tau\left(t_{k}\right)\right\}=\left\{2 d \eta\left(t_{k}\right)<\tau\left(t_{k}\right)\right\}$. Then the result follows from the corollary, on taking $\varepsilon=1 / 2$. 


\section{Lower Bound for the Hausdorff Measure}

We wish now to show that the Hausdorff measure induced by $\varphi$,

$$
\varphi-m(E)=\lim _{\delta>0} \inf \left\{\sum_{1}^{\infty} \varphi\left(d\left(J_{i}\right)\right): E \subset \bigcup_{1}^{\infty} J_{i}, d\left(J_{i}\right)<\delta\right\},
$$

is positive, possibly infinite, on certain sets $E$ of the form $Z \cap[a, b]$, or equivalently, as noted at the end of section 3 , on sets $E=Q \cap[a, b]$. Direct application of the definition would require the determination of positive $\varepsilon, \delta$ such that any covering of $E$ by sets $J_{i}$ of diameters $d\left(J_{i}\right)<\delta$ has the property that $\sum \varphi\left(d\left(J_{i}\right)\right) \geqq \varepsilon$. This is difficult, as it is not sufficient, for instance, to consider coverings of $E$ by finitely many intervals all of the same length. One may get a more economical covering by using intervals of widely differing lengths.

We therefore resort to a method first used in [5] for showing that the Hausdorff measure of some random sets is positive. This involves "spreading" a set function defined on all Borel sets "uniformly" on $E$ and then analyzing it by the following lemma, which is a modified (and simpler) version of Lemma 3 of [17].

Lemma 4. Suppose that $F$ is a completely additive measure defined on the real Borel sets and that $E$ is a Borel set such that for each $x \in E$

Then

$$
\limsup _{h \rightarrow 0} \frac{F[x, x+h]}{\varphi(h)} \leqq k<\infty .
$$

$$
k \varphi-m(E) \geqq F(E) .
$$

Proof. Since $F$ must be a regular measure in the sense that

$$
F(E)=\sup \{F(K): K \subset E, K \text { compact }\}
$$

we may assume that $E$ is compact. Let $\delta>0$ and put

$$
H_{q}=\left\{x \in E: \sup _{0<h<1 / q} F[x, x+h] / \varphi(h) \leqq k+\delta\right\} .
$$

Then $H_{q}$ is closed and $E=\bigcup_{q=1}^{\infty} H_{q}$. If $\varphi-m(E)=\infty$ there is nothing to prove. If $\varphi-m(E)<\infty$ then $\varphi-m\left(H_{q}\right)<\infty$ and we can find a covering of $H_{q}$ by intervals $J_{i, q}$ whose lengths are less than $1 / q$ and such that

$$
\sum_{i=1}^{\infty} \varphi\left(d\left(J_{i, q}\right)\right)<\varphi-m\left(H_{q}\right)+\delta .
$$

We may assume that the intervals $J_{i, q}$ are closed and that their left endpoints lie in $H_{q}$. Then

so that

$$
F\left(J_{i, q}\right) \leqq(k+\delta) \varphi\left(d\left(J_{i, q}\right)\right)
$$

$$
F\left(H_{q}\right) \leqq \sum F\left(J_{i, q}\right)<(k+\delta)\left(\varphi-m\left(H_{q}\right)+\delta\right) .
$$

Since $H_{q}$ is increasing in $q$ and $E=\bigcup H_{q}$ this gives

$$
F(E) \leqq(k+\delta)(\varphi-m(E)+\delta),
$$

and the lemma is established by letting $\delta$ decrease to zero. 
We now use Lemma 4 to prove that

$$
p-m(Q \cap[0, \tau(\mathbf{1})])
$$

is almost surely positive. The appropriate set function $F(E)=F^{\prime}(E)$ to spread over the set $Q(\omega) \cap[0, \tau(1, \omega)]$ is the one induced by the local time $A(t, \omega)$. That is, we define a measure $F_{\omega}(E)$ of Borel sets $E$ by setting $F_{\omega}[t, t+h]$ equal to the difference $A(t+h, \omega)-A(t, \omega)$ and extending the definition from intervals to Borel sets in the usual way. Since $A$ increases only at the points of $Q$, and $A, \tau$ are inverse to each other, it follows that $F_{\omega}$ is carried on $Q(\omega)$ and that

$$
F_{\omega}[0, \tau(t, \omega)]=F_{\omega}(Q(\omega) \cap[0, \tau(t, \omega)])=t ;
$$

therefore if $E$ is a Borel set then $F(Q \cap \tau(E))$ is simply its Lebesgue measure.

Now let $\Gamma$ denote the set of pairs $(t, \omega) \in[0,1] \times \Omega$ at which

$$
\limsup _{h \rightarrow 0} \frac{F_{\omega}[\tau(t, \omega), \tau(t, \omega)+h]}{\varphi(h)} \leqq d^{-\beta} .
$$

By the strong Markov property and Theorem 4 applied at 0 we see that each $t$-section of $T$ has probability 1 . Then by Fubini's theorem almost every $\omega$-section $\Delta=\Delta(\omega)$ has Lebesgue measure 1. Then Lemma 4 implies that almost surely

$$
\varphi-m(Q \cap[0, \tau(1)]) \geqq \varphi-m(Q \cap \tau(\Delta)) \geqq d^{\beta} F(Q \cap \tau(\Delta))=d^{\beta}>0 .
$$

\section{Upper Bound for the Hausdorfi Measure}

In order to show that $\varphi-m(Q \cap[0,1])<\infty$ we have to provide an economical $p$-covering of the set; it is easy to see that it is not adequate to consider coverings by intervals all of the same length. In fact, one can modify the arguments of ITO-McKkaN [10] p. 50 for the Wiener process to deduce that using coverings by equal intervals will only give finite measure with respect to the measure function $h^{\beta}$. In order to make use of coverings of a more general nature we modify the arguments first used in [21]. To this end we need a lemma which will enable us to deduce that we have not been too wasteful in our final covering. This means that we do not want to use intervals that overlap too much. Consider the collection $\Lambda_{h}$ of intervals $\left[(j-1) / 2^{h},(j+1) / 2^{h}\right), j=1,2, \ldots$ Any interval of length $l<2^{-h}$ can be covered by one of the intervals of $\Lambda_{h}$. Then any interval $I$ is contained in an interval of $\bigcup_{h_{0}}^{n} \Lambda_{h}$ of length at most four times that of $I$, providing that the length of $I$ is a number in the interval $\left[2^{-n-1}, 2^{-h_{0}}\right)$.

We make precise the fact that the intervals of $\Lambda_{h}$ are "almost nested".

Lemma 5. If $E=\bigcup_{j=1}^{m} I_{j}$, where each $I_{j}$ is an interval of $\Lambda_{h}$ for some $h$ between $h_{0}$ and $n$, then it is possible to find a subset $\left\{j_{r}\right\}$ such that $E=\bigcup I_{j_{r}}$ and no point of $E$ is in more than two of the intervals $I_{j_{r}}$.

This can be proved by the same argument used to give Lemma 1 of [21].

We proceed now to the construction of the covering.

Given $\delta>0$ choose $h_{0}$ so that $2^{-h_{0}}<\min \left(\delta / 2, u_{m_{0}}\right)$, where $u_{k}$ and $m_{0}$ are as in Corollary 2. Choose $m$ such that $u_{m}<2^{-h_{0}}$. Given $n$, let $M_{n}$ be the largest 
integer $k$ such that $u_{k} \geqq 2^{-n-1} ; n$ should be taken large enough so that $n>h_{0}$ and $M_{n} \geqq 2 \mathrm{~m}$. It is not difficult to check that for suitable positive constants $c_{18}, c_{19}$ we have $M_{n} \geqq c_{18} n^{c_{19}}$ when $n$ is sufficiently great.

For such a fixed $n$ consider the intervals $I_{j, n}=\left[(j-1) 2^{-n}, j 2^{-n}\right)$. We say that $I_{j, n}$ is $b a d$ for the sample point $\omega$ if (i) $Q(\omega)$ meets $I_{j, n}$ and (ii) there is no interval $[a, b)$ of $\bigcup_{h_{0}}^{n} \Lambda_{h}$ such that $[a, b)$ contains $I_{j, n} \cap Q(\omega)$ and

$$
\frac{A(b, \omega)-A(a, \omega)}{\varphi(b-a)} \geqq d^{\prime} / 8,
$$

$d^{\prime}$ as in Lemma 3. All other intervals $I_{j, n}$ are said to be good. If $I_{j, n}$ is good then either $Q \cap I_{j, n}$ is void or it can be covered by an interval $[a, b)$ of $\bigcup_{h_{0}}^{n} \Lambda_{h}$ with $\{A(b)-A(a)\} / \varphi(b-a) \geqq d^{\prime} / 8$. We complete the covering of $Q$ by taking $I_{j, n}$ itself to cover the set $Q \cap I_{j, n}$ when the interval is bad. Then all intervals of the covering have length less than $\delta$.

We now show that the contribution to the covering from bad intervals is small. If $Q \cap I_{j, n}$ is not void, let $s_{j}$ be the least point of $Q$ not less than $(j-1) / 2^{n}$. Let $B_{k, j}$ be the event that $\left\{A\left(s_{j}+u_{k}\right)-A\left(s_{j}\right)\right\} / \varphi\left(u_{k}\right)<d^{\prime} / 2$, for $k=m$, $m+1, \ldots, M_{n}$. By Lemma 3 and the strong Markov property we know that $\bigcap_{k=m}^{M_{n}} B_{k, j}$ has a probability at most $\exp \left(-c_{17} M_{n}^{c_{16}}\right) \leqq \exp \left(-c_{20} n^{c_{21}}\right)$. The length $u_{k}$ of the interval $\left[s_{j}, s_{j}+u_{k}\right.$ ) is a number in the interval $\left[2^{-n-1}, 2^{-h_{0}}\right)$. Hence $\left[s_{j}, s_{j}+u_{k}\right)$ can be covered by an interval $[a, b)$ of $\bigcup_{h_{0}}^{n} \Lambda_{h}$ such that
$b-a \leqq 4 u_{k}$.

Suppose that $\omega$ is in the complement of the set $\bigcap_{m}^{M_{n}} B_{k, j}$. Then there is at least one $k$ with

$$
\frac{A\left(s_{j}+u_{k}\right)-A\left(s_{j}\right)}{\varphi\left(u_{k}\right)} \geqq d^{\prime} / 2 .
$$

Covering that interval $\left[s_{j}, s_{j}+u_{k}\right)$ as just indicated we have

$$
\frac{A(b)-A(a)}{\varphi(b-a)} \geqq \frac{A\left(s_{j}+u_{k}\right)-A\left(s_{j}\right)}{\varphi\left(4 u_{k}\right)} \geqq d^{\prime} / 8
$$

since $\varphi(4 h)<4 \varphi(h)$ when $h$ is small. Thus the interval $I_{j, n}$ is good. Therefore

$$
P\left\{I_{j, n} \text { is bad }\right\} \leqq P\left\{Q \text { meets } I_{j, n}\right\} \exp \left(-c_{20} n^{c_{21}}\right)<c_{22} j^{\beta-1} \exp \left(-c_{20} n^{c_{21}}\right)
$$

on using Lemma 1 .

Now let $T_{n}$ denote the number of bad intervals $I_{j, n}$ with $1 \leqq j \leqq 2^{n}$. It follows that

$$
E\left\{T_{n}\right\} \leqq c_{22}\left[\exp \left(-c_{20} n^{c_{21}}\right)\right] \sum_{1}^{n} j^{\beta-1} \leqq c_{23} 2^{n \beta} \exp \left(-c_{20} n^{c_{21}}\right) .
$$

The covering of bad intervals must make a contribution

$$
\Sigma_{n}^{\prime}=T_{n} 2^{-n \beta}[\log (n \log 2)]^{1-\beta},
$$

whose expectation is majorized by an expression of the form

$$
\chi_{n}=c_{24}(\log n)^{1-\beta} \exp \left(-c_{20} n^{c_{21}}\right) .
$$


For any $\varepsilon>0$ we have then $P\left\{\Sigma_{n}^{\prime}>\varepsilon\right\}<\chi_{n} / \varepsilon$. Set $\varepsilon=1 / n$ and allow $n$ to vary; applying the Borel-Cantelli lemma we deduce that with probability 1 there exists an integer $n_{0}$ such that, for $n>n_{0}$

$$
\Sigma_{n}^{\prime}<1 / n
$$

so the contribution of the bad intervals is negligible.

For each good interval which contains a point of $Q$ we choose an interval $[a, b)$ in $\bigcup_{h_{0}}^{n} \Lambda_{h}$ such that $\{A(b)-A(a)\} / \varphi(b-a) \geqq d^{\prime} / 8$. This gives a finite collection of intervals to which Lemma 5 can be applied. We obtain a set of the form $\cup\left[a_{i}, b_{i}\right)$ which still covers the good intervals $I_{j, n}$ but covers none of them more than twice. For this covering

$$
\sum\left(A\left(b_{i}\right)-A\left(a_{i}\right)\right)<2(A(e)-A(0))
$$

where $e$ is $\sup b_{i}<1+2^{-h_{0}}$.

Hence

$$
\sum^{\prime \prime} \varphi\left(b_{i}-a_{i}\right) \leqq c_{25} A\left(1+2^{-h_{0}}\right), \quad c_{25}=16 / d^{\prime},
$$

where $\sum$ " denotes the resultant covering of the good intervals. Thus, combining (10) and (11) we obtain a finite covering of $Q \cap[0,1]$ for each $n>n_{0}$ such that

$$
\sum \varphi\left(d\left(J_{i}\right)\right) \leqq c_{25} A\left(1+2^{-h_{0}}\right)+\frac{1}{n} .
$$

Letting $\delta \rightarrow 0, h_{0}$ tends to infinity; using the continuity of $A$ we obtain

$$
\varphi-m(Q \cap[0,1]) \leqq c_{25} A(1)
$$

with probability 1 . Since $A(1)$ is finite almost surely, the proof is complete.

\section{Proof of Theorem 1}

The results of the preceding two sections show that with probability one the $\varphi$-measure of $Q \cap[0, \tau(1)]$ is positive, while that of $Q \cap[0,1]$ is finite. The same arguments evidently apply with $[0, \tau(1)]$ replaced by $[0, \tau(1 / N)]$, or with $[0,1]$ replaced by $[0, N]$, where $N$ is any positive integer. Then it follows that almost surely the random function

$$
f(t)=p-m(Q \cap[0, \tau(t)])
$$

is finite and positive for all $t>0$. It is clear that the process $f(t)$ has the following properties: it has stationary independent increments; it has continuous paths almost surely; it is monotonic. The first two imply ([13], Theorem V2.3) that for suitable $\mu, \sigma$ we may write $f(t)=\mu t+\sigma w(t)$, where $w(t)$ is a Wiener process with mean zero and variance $t$. Monotonicity then shows that $\sigma=0$. Positivity of $f$ implies that $\mu=c_{1}>0$. Thus with probability $1, \varphi-m(Q \cap[0, \tau(t)])=c_{1} t$ for all $t \geqq 0$. Replacing $t$ by $A(t)$ we obtain the desired conclusion. All of this has, of course, been carried through under the assumption $b=1$. But, as mentioned at the end of section 3 , the general case is obtained on replacing $c_{1}$ by $b c_{1}$.

It follows, by a further application of the strong Markov property, that if

$$
Z_{x}=\{t: X(t)=x\}
$$


is the occupation time set of the point $\{x\}$ for the process $X(t)$ then for each fixed $x$ we have

$$
\varphi-m\left(Z_{x} \cap[0, t]\right)=b c_{1} L(x, t) \text { for all } t \geqq 0
$$

almost surely. It becomes of some interest to ask whether (12) is almost surely true simultaneously for all $x$ and all $t \geqq \mathbf{0}$. We have not been able to settle this question.

It would also be of interest to extend our results to more general processes with independent increments - see [2].

\section{References}

[1] Blumenthal, R. M., and R. K. Getoon: Sample functions of stochastic processes with stationary independent increments. J. Math. Mech. 10, 493-516 (1961).

[2] - - : The dimension of the set of zeros and the graph of a symmetric stable process. Illinois J. Math. 6, 308-316 (1962).

[3] - - Local times for Markov processes. Z. Wahrscheinlichkeitstheorie verw. Geb. 3, $50-74$ (1964).

[4] BoyLAN, E. S.: Local times for a class of Markov processes. Illinois J. Math. 8, 19-39 (1964).

[5] CresielsKi, Z., and S. J. TAYLOR: First passage times and sojourn times for Brownian motion in space and the exact Hausdorff measure of the sample path. Trans. Amer. math. Soc. 108, $434-450$ (1962).

[6] Dynkin, E. B.: Some limit theorems for sums of independent random variables with infinite mathematical expectation. Izvestija Akad. Nauk. SSSR, Ser. Mat. 19, 247 to 266 (1955).

[7] FRISTEDT, B.: The behavior of increasing stable processes for both small and large times. J. Math. Mech. 13, 849-856 (1964).

[8] GETOOR, R. K.: Additive functionals and excessive functions. Ann. math. Statistics 36, $409-422(1965)$.

[9] Hunt, G. A.: Markor processes and potentials I., II, III. Mlinois J. math. 1, 44-93 $316-369(1957)$ and $2,151-213(1958)$.

[10] Iтo, K., and H. P. MoKeAv: Diffusion processes and their sample paths. Berlin-Heidelberg-New York: Springer 1965.

[11] KESTEN, H.: An iterated logarithm law for local time. (To appear.)

[12] Kнтлснтr, A.: Sur la croissance locale des processus stochastique homogenes à àcroissements independants. Tzvestija Akad. Nauk. SSSR, Ser. Mat. , 487-508 (1939).

[13] KRICKEBERG, K.: Probability theory. Reading, Mass.: Addison-Wesley 1965.

[14] Lóvy, P.: Processus stochastiques et mouvement Brownien. Paris 1948.

[15] Linsm, Yu. V.: On stable probability laws with exponent less than one. Doklady Akad. Nauk. SSSR, n. Ser. 94, 619-621 (1954).

[16] Pollakd, H.: The representation of $e^{-x^{\lambda}}$ as a Laplace integral. Bull. Amer. math. Soc. 52, 908-910 (1946).

[17] Rogers, C. A., and S. J. TAXLOR: Functions continuous and singular with respect to a Hausdorff measure. Mathematika, London 8, 1-31 (1961).

[18] Sronoнod, A. V.: Asymptotic formulas for stable distribution laws. Selected translations in mathematical statistics and probability, 1, 157-162 (1961); Doklady Akad. Nauk. SSSR, n. Ser. 98, 731-734 (1954).

[19] Stone, C. J.: The set of zeros of a semi-stable process. Illinois J. Math. 7, 631-637 (1963).

[20] TAYLOR, S. J.: The $\alpha$-dimensional measure of the graph and the set of zeros of a Brownian path. Proc. Cambridge philos. Soc. 51, 265-274 (1955).

[21] - : The exact Hausdorff measure of the sample path for planar Brownian motion. Proc. Cambridge philos. Soc. 60, 253-258 (1964).

[22] Trotтm, H. F.: A property of Brownian motion paths. Illinois J. Math. 2, 425-433 (1958). 